Bangladesh Journal of Neuroscience 2012; Vol. 28 (2) : 96-101

\title{
Comparison of Clinical Diagnosis of Stroke with Computed Tomographic Scan of the Brain
}

\author{
SIRAJEE SHAFIQUL ISLAM ${ }^{1}$, AMINUR RAHMAN $^{2}$, MD. MANZUR ALAHI $^{3}$, MD. AHMED ALI $^{3}$ \\ MD. KAFILUDDIN ${ }^{3}$, MD. PERVEZ AMIN ${ }^{4}$, QUAMRUDDIN AHMAD ${ }^{5}$, DEWAN MD ELYAS ${ }^{6}$, \\ MD .ZIAUL KARIM ${ }^{7}$, MOHAMMAD SHAHEEN WADUD ${ }^{8}$, MD. RAFIQUL ISLAM ${ }^{9}$
}

\begin{abstract}
:
Background and purpose: Stroke is a leading cause of mortality and morbidity in both developed as well as developing countries. The clinical presentation of stroke depending on the site and extent of lesions. For the management purpose it is important to know whether we are dealing with a bleed or an infarct . Methodology: Computed Tomography (CT scan) is available most of the tertiary level hospitals in Bangladesh. This study was carried out to compare clinical diagnosis of stroke with Computed tomography (CT) scan findings in ascertaining the type of stroke (hemorrhagic or ischemic). Materials and methods: This cross-sectional comparative study was conducted in the Department of Neurology, Rajshahi Medical College Hospital during the period of January 2010 to December 2010. Total 200 stroke patients were selected by purposive sampling technique on the basis of inclusion and exclusion criteria as the study sample. . CT brain scan was done for all the patients. The clinical diagnosis was compared with the results of CT scan and performance test was done. Results: Clinically 67 patients were diagnosed as hemorrhagic stroke and 133 patients were diagnosed as ischemic stroke. Out of these 67 hemorrhagic patients CT scan revealed that 56 patients had intracerebral hemorrhage, 5 had infarct, 4 had subarachnoid hemorrhage and 2 had space occupying lesions in the brain. Out of these 133 ischemic patients CT scan revealed that 119 patients had infarction, 6 had intracerebral hemorrhage and 8 had space occupying lesions in the brain. The sensitivity, specificity, positive predictive value, negative predictive value and accuracy of clinical diagnosis of hemorrhagic stroke were 90.32\%, 92.03\%, 83.58\%, 92.02\% and 91.5\% respectively. The sensitivity, specificity, positive predictive value, negative predictive value and accuracy of clinical diagnosis of ischemic stroke were 95.96\%, 81.58\%, 89.47\%, 92.53\% and 90.5\% respectively. Conclusion: The diagnosis of stroke in clinically with high accuracy, but perform a CT scan will help to confirm and differentiate to type stroke. Thus CT scan should be done in all cases stroke to specify the diagnosis.
\end{abstract}

Key Words: Stroke, Computed Tomography Scan.

Introduction:

Cerebrovascular diseases include some of the most common and devastating disorders: ischemic stroke, hemorrhagic stroke, and cerebrovascular anomalies such as intracranial aneurysms and arterio-venous malformations. They cause 200,000 deaths each year in the United States and are a major cause of disability. The incidence of cerebrovascular diseases increases with age, and the number of strokes is projected to increase as the elderly population grows, with a doubling in stroke deaths in the United States by $2030^{1}$. Stroke

1. Registrar, Dept of Interventional Neurology. National Institute of Neurological Sciences, Dhaka.

2. Registrar, Dept of Neurology Sir Salimullah Medical College Hospital. Dhaka

3. Assistant Prof. Dept. of Neurology, Rajshahi Medical College, Rajshahi

4. Medical Officer, Dept. of Neurology, Rajshahi Medical College Hospital, Rajshahi

5. Professor and Head, Dept. of Neurology, Rajshahi Medical College Hospital, Rajshahi

6. Indoor. Medical Officer, Department of Neurology, National Institute of Neurosciences\& Hospital, Dhaka.

7. Assistant Prof. Dept. of Respiratory Medicine, National Institute of Chest Diseases, Dhaka.

8. Resident, Dept of Neurology Sir Salimullah Medical College Hospital. Dhaka

9. Professor, Department of Neurology, Bangabandhu sheikh Mujib Medical University, Dhaka 
is a one of the leading cause of mortality and morbidity in both developed as well as developing countries like Bangladesh. The clinical picture and epidemiology is variable depending on the site and extent of lesions ${ }^{2}$.

Stroke is defined by this abrupt onset of a neurologic deficit that is attributable to a focal vascular cause. Thus, the definition of stroke is clinical, and laboratory studies including brain imaging are used to support the diagnosis. The clinical manifestations of stroke are highly variable because of the complex anatomy of the brain and its vasculature ${ }^{1}$.

The differentiation of cerebral infarction and cerebral haemorrhage is the most important first step in the management of acute stroke, because clinical management of the two disorders differs substantially ${ }^{3}$. The distinction between cerebral infarction $(\mathrm{Cl})$ and intracerebral haemorrhage $(\mathrm{ICH})$ is now seen as crucial in determining prognosis and acute care and to promote secondary stroke prevention. Certain drugs or procedures may benefit patients with $\mathrm{Cl}$ but they are potentially dangerous in those with haemorrhage. The classic clinical features of $\mathrm{ICH}$ (sudden onset with severe headache, vomiting, rapid deterioration of consciousness, lack of previous transient events) are frequent and reasonably specific only in massive haemorrhage. However, distinction between $\mathrm{Cl}$ and small or superficial haemorrhages using these simple criteria seems to be considerably more difficult. To deal with this problem, clinical scoring systems including several variables have been constructed and are considered to be more accurate than unstructured clinical diagnosis as usually made in clinical practice. Instruments commonly used for this purpose include those from the Guys Hospital, from Siriraj Hospital and from the Grenoble University Center ${ }^{4}$.

The introduction of computed tomography (CT) to clinical practice has had a great impact on our knowledge of cerebrovascular disorders, and cerebral CT has become the most commonly used primary radiologic investigation for stroke. Cerebral CT has shown that the prognosis of intracerebral hemorrhage $(\mathrm{ICH})$ is not as poor as was supposed when small hemorrhages were often undiagnosed or misdiagnosed as ischemic events, and it has changed the order of diagnostic procedures for stroke. Furthermore, in differentiating ischemic infarcts from hemorrhagic lesions, cerebral CT has proved to be of crucial importance for therapeutic considerations, particularly anticoagulant treatment ${ }^{5}$.

The radiologic diagnosis of stroke requires accurate detection and appropriate interpretation of relevant imaging findings; both detection and interpretation may be influenced by knowledge of the patient's presentation. Availability of a clinical history indicating that early stroke is suspected significantly improves the sensitivity for detecting strokes on non-contrast CT without reducing specificity. Whenever possible, relevant clinical history should be made available to physicians interpreting emergency CT scans of the head ${ }^{6}$. Even then treatment for acute ischemic stroke is now being carried out before brain images have become positive, increasing reliance is being placed on early clinical assessment ${ }^{7}$. For faster access to acute stroke treatments, quicker transfer to stroke units and earlier secondary stroke prevention, stroke and TIA patients need to be accurately identified in the emergency department ${ }^{8}$.

\section{Materials and Methods:}

This cross-sectional comparative study was conducted in the Department of Neurology, Rajshahi Medical College Hospital (RMCH) during the period of January 2010 to December 2010. Two Hundred suspected acute stroke patients were selected by purposive sampling technique with inclusion criteria were, 1)Patient presenting with acute onset focal neurological deficits , 2)Neurological deficit lasting for more than 24 hours and 3)Age: 18 years or above and exclusion criteria were 1) Recurrent stroke. 2) Stroke duration of more than 14 days because of the possibility of missing an intracerebral haemorrhage. 3) Stroke associated with hematological conditions like leukemia, polycythaemia and 4) Metabolic disorders like hypoglycemia.

Data will be collected by face-to-face interview, physical examination and investigations in a data collection sheet.. On admission detailed history and thorough clinical examination including neurological assessment were carried out. Emphasis was given on risk factors especially hypertension, coronary artery disease, atrial fibrillation, rheumatic heart disease, peripheral vascular disease, smoking and diabetes mellitus etc. The clinical diagnosis of type 
of stroke was made on the basis of the neurological history and signs. Those patients who presented with sudden onset of coma, rapid deterioration of neurological state, severe headache and vomiting and neck stiffness along with hypertension were considered to be suffering from hemorrhagic stroke. Patients who presented with sudden onset of lateralizing signs especially in the presence of atrial fibrillation, rheumatic heart disease, recent myocardial infarction and carotid bruit were considered to be suffering from ischemic stroke. In addition to routine investigation s, blood sugar, lipid profile, ECG and in some selected echocardiography were performed. All patients had CT scan brain were done in all patients from the department of radiology. Then the clinical diagnosis was compared with the results of CT scan and precision of clinical diagnosis was ascertained.

Statistical analyses related with this study were performed by using of SPSS 13 package program The comparisons between patients with hemorrhagic and ischemic stroke with the student t test for normally distributed continuous variables and $\chi^{2}$ tests for dichotomous variables. Test of performance were done to detect the sensitivity, specificity, positive predictive value, negative predictive value and accuracy. Ethical clearance is taken from ethical committee of $\mathrm{RMCH}$. Every patient and/or responsible family member will were asked for informed consent about the procedure and the study goal.

\section{Results and Observation:}

Table-I

Demographic status of the patients

\begin{tabular}{lccc}
\hline & $\begin{array}{c}\text { Hemorrhagic } \\
(\mathrm{n}=67)\end{array}$ & $\begin{array}{c}\text { Infarction } \\
(\mathrm{n}=133)\end{array}$ & $\mathrm{p}$ value \\
\hline $\begin{array}{l}\text { Age of the patients } \\
\text { (Mean } \pm \text { SD) }\end{array}$ & $57.61 \pm 8.3$ & $56.30 \pm 9.70$ & $.346^{\mathrm{NS}}$ \\
Sex of the patients, & & & \\
frequency (\%) & & & \\
Male & $35(52.2 \%)$ & $63(47.4 \%)$ & $.515^{\mathrm{NS}}$ \\
Female & $32(47.8 \%)$ & $70(52.6 \%)$ & \\
\hline
\end{tabular}

The $p$ value of categorical variables were obtained by chi-square test and continuous variables by Student't' test.

The Table-I shows, the mean ( \pm SD) age of the patients with hemorrhagic stroke was $57.61( \pm 8.3)$ years and the mean $( \pm S D)$ age of the patients with ischemic stroke was $56.3( \pm 9.7)$ years. The difference of mean age between two groups were not statistically significant $(p>0.05)$. Among the patients with hemorrhagic stroke 35(52.2\%) were male and $32(47.8 \%)$ were female. Among the patients with ischemic stroke $63(47.4 \%)$ were male and $70(52.6 \%)$ were female. The difference of gender between two groups were not statistically significant $(p>0.05)$.

Table-II

Clinical diagnosis of type of stroke

\begin{tabular}{lcc}
\hline $\begin{array}{l}\text { Clinical diagnosis } \\
\text { of type of stroke }\end{array}$ & Frequency & Percent \\
\hline Hemorrhagic & 67 & 33.5 \\
Ischemic & 133 & 66.5 \\
\hline
\end{tabular}

Table III

Cross tabulation of clinical type of stroke and diagnosis by CT scan

\begin{tabular}{lcccc}
\hline & & \multicolumn{2}{c}{ Clinically diagnosed type of stroke } & Total \\
\cline { 2 - 4 } & & Hemorrhagic & Infarction & \\
\hline Diagnosis by CT Scan & Hemorrhage & $56(83.6 \%)$ & $6(4.5 \%)$ & $62(31.0 \%)$ \\
of Brain & Infarction & $5(7.5 \%)$ & $119(89.5 \%)$ & $124(62.0 \%)$ \\
& SAH & $4(6.0 \%)$ & $0(0.0)$ & $4(2.0 \%)$ \\
& SOL & $2(3.0 \%)$ & $8(6.0 \%)$ & $10(5.0 \%)$ \\
\hline & Total & $67(100 \%)$ & $133(100 \%)$ & 200 \\
\hline
\end{tabular}

Table-IV

Performance of clinical diagnosis in diagnosing hemorrhagic stroke

\begin{tabular}{llccc}
\hline Diagnostic Modality & & \multicolumn{2}{c}{ Diagnosis by CT Scan } & Total \\
\cline { 3 - 4 } & & Hemorrhagic & Other than hemorrhage & \\
\hline Clinical type of & Hemorrhage & $\mathrm{a}=56$ & $\mathrm{~b}=11$ & $\mathrm{a}+\mathrm{b}=67$ \\
Stroke & Other than hemorrhage & $\mathrm{c}=6$ & $\mathrm{~d}=127$ & $\mathrm{c}+\mathrm{d}=133$ \\
\hline & Total & $\mathrm{a}+\mathrm{c}=62$ & $\mathrm{~b}+\mathrm{d}=138$ & $\mathrm{~N}=\mathrm{b}+\mathrm{c}+\mathrm{d}=200$ \\
\hline
\end{tabular}


Table II, shows that among the 200 patients $67(33.5 \%)$ patients were diagnosed as hemorrhagic stroke and 133(66.5\%) patients were diagnosed as infarctive stroke

From table III, it was revealed that, clinically 67 patients were diagnosed as hemorrhagic stroke. Out of these 67 patients CT scan revealed that 56 (83.6\%) patients had intracerebral hemorrhage, 5 had infarct, 4 had subarachnoid hemorrhage and 2 had space occupying lesions in the brain. Clinically 133 patients were diagnosed as having infarction. Out of these 133 patients, CT scan revealed that 119 patients had infarction, 6 had intracerebral hemorrhage and 8 had space occupying lesions in the brain.

The Table IV shows that the sensitivity, specificity, positive predictive value, negative predictive value and accuracy of clinical diagnosis of hemorrhagic stroke were $90.32 \%, 92.03 \%, 83.58 \%, 92.02 \%$ and $91.5 \%$ respectively.

\section{Discussion:}

The present study (Table I) revealed that the mean $( \pm S D)$ age of the patients with hemorrhagic stroke was $57.61( \pm 8.3)$ years and the mean $( \pm S D)$ age of the patients with ischemic stroke was $56.3( \pm 9.7)$ years. Among the patients with hemorrhagic stroke 35(52.2\%) were male and 32(47.8\%) were female. Among the patients with ischemic stroke 63(47.4\%) were male and $70(52.6 \%)$ were female. The findings of the present study were supported by Baloch et al (2009), studied in the Liaquat University Hospital, Hydrabad, India9. Among a total of 110 patients Baloch et al (2009) found that 60 (54.5\%) were males and 50 (45.5\%) were females. Age of patients ranged 22-84 years with mean ( $\pm S D$ ) age of $53( \pm 5)$ years. In the present study (Table II) among the 200 patients $67(33.5 \%)$ patients were clinically diagnosed as hemorrhagic stroke and 133(66.5\%) patients were diagnosed as ischemic stroke. Out of 67 clinically diagnosed hemorrhagic stroke patients CT scan revealed that 56 patients had intracerebral hemorrhage, 5 had infarct, 4 had subarachnoid hemorrhage and 2 had space occupying lesions in the brain. Out of 133 clinically diagnosed ischemic stroke patients CT scan revealed that 119 patients had infarction, 6 had intracerebral hemorrhage and 8 had space occupying lesions in the brain.

Among a total of 110 patients Baloch et al (2009) found that on clinical ground cerebral infarction was suspected in $89(80.9 \%)$ and cerebral hemorrhage in $21(19.1 \%)$ patients ${ }^{9}$. Based on clinical features, $62 \%$ of patients were categorized as cerebral infarcts and $30 \%$ and $8 \%$ were diagnosed as intracerebral and subarachnoid hemorrhage, respectively. CT scanning revealed that $8 \%$ of patients with clinical diagnosis of stroke were having other cerebral pathology (4\% hydrocephalus, $4 \%$ cerebral tumor) ${ }^{10}$.

Khan and Rahman (2005) found that CT scan brain showed $60 \%$ cerebral infarction, $27 \%$ intracerebral hemorrhages, $9 \%$ space occupying lesion and $4 \%$ hemorrhagic infarct ${ }^{11}$.Siddique et al (2009) conducted a study among 100 stroke patients in Chittagong Medical college hospital of Bangladesh. Ischemic stroke was found in $80 \%$ cases and hemorrhagic stroke was found in $20 \%$ cases ${ }^{12}$. Whiteley et al (2011)o conducted a cross-sectional study among 405 patients with suspected stroke, of whom $285(70 \%)$ had symptoms owing to a final diagnosis of probable or definite acute cerebrovascular diseases, and 120 (30\%) owing to other illness ${ }^{8}$.

Hill (2010) stated that ischaemic stroke is the most common stroke type, comprising $65-85 \%$ of all stroke, varying by location in the world. Two main haemorrhagic forms of stroke are intracerebral hemorrhage $(\mathrm{ICH})$ and subarachnoid haemorrhage ${ }^{13}$.

Wardlaw (1994) observed that outside hospital about $80 \%$ of strokes are cerebral infarcts, $10 \%$ are primary intracerebral haemorrhages, $5 \%$ are subarachnoid haemorrhages, and about $5 \%$ are of uncertain cause. Wardlaw (1994) also observed that in hospital only $60-75 \%$ of patients with stroke have a cerebral infarct ${ }^{14}$.

Naik et al (2006) stated that there are four major types of stroke, eg: cerebral infarction, intracerebral haemorrhage $(\mathrm{ICH})$, primary subarachnoid hemorrhage (SAH) and venous occlusion. Cerebral infarction is due to significantly diminished blood flow to all parts of the cerebral 
hemisphere (global) or selected areas (regional or focal) of the brain. Naik et al (2006) found that among the stroke subtypes, cerebral ischernia and infarction constitute about $85-90 \%$ of the total stroke subtypes in western countries with only about 10$15 \%$ patients with cerebral haemorrhage. But contrary to the western population, hemorrhagic stroke constitutes a larger percentage of stroke subtypes on this side of the globe as seen in countries like Japan and China probably because of poorly controlled hypertension ${ }^{15}$.

Sotaniemi, Pyhtinen, Myllyla (1990) studied 1,191 consecutive patients with acute cerebrovascular disease and found that computed tomography scan revealed hemorrhagic lesion in $33.8 \%$, ischemic in $66.2 \%$ and a significant non-stroke abnormality in $3.1 \%$. They emphasized that both careful neurologic assessment and a policy of early computed tomography scan are of crucial importance in the diagnosis of stroke and for therapeutic consideration ${ }^{5}$.

In the current study (Table III), the sensitivity, specificity, positive predictive value, negative predictive value and accuracy of clinical diagnosis of hemorrhagic stroke were 90.32\%, 92.03\%, $83.58 \%, 92.02 \%$ and $91.5 \%$ respectively. In case of ischemic

stroke (Table IV) the sensitivity, specificity, positive predictive value, negative predictive value and accuracy were 95.96\%, 81.58\%, 89.47\%, 92.53\% and $90.5 \%$ respectively.

To develop a simple, reliable, and safe diagnostic tool for acute stroke syndromes in a setting where computerized brain scanning was not readily available, The Siriraj Stroke Scale was developed in Mahidol University, Bangkok ${ }^{16}$ and was calculated a score above 1 indicates supratentorial intracerebral haemorrhage, while a score below -1 indicates infarction. The score between 1 and -1 represents an equivocal result needing a computerized brain scan or probability curve to verify the diagnosis. In the validation study of the Siriraj stroke score the diagnostic sensitivities of the score for cerebral haemorrhage and cerebral infarction were $89.3 \%$ and $93.2 \%$ respectively, with an overall predictive accuracy of $90.3 \% .^{17,18}$ To improve the clinical diagnosis of stroke in the emergency department, Nor, et $\mathrm{al}^{2}{ }^{19}$ formulated the ROSIER scale (Recognition of Stroke in the Emergency Department). Rapid assessment and triage by paramedics had achieved a consistent diagnostic accuracy of between $80 \%$ and $95 \%$ and so, this study is consistent with our study ${ }^{19}$.

\section{Conclusion:}

The diagnosis of strokes can be done clinically with high accuracy, but a CT scan of brain will help to confirm the diagnosis and differentiate the types stroke. Thus CT scan should be done in all of cases strokes to confirm the diagnosis.

\section{References:}

1. Fauci AS, Kasper, Braunwald E . Cerebrovascular Diseases. Harrison's priciples of Internal Medicine. $17^{\text {th }}$ ed. New York, Delhi: Mc Graw Hill 2008;364:2513

2. Clarke CRA. Cerebrovascular disease and stroke. In: Kumar P and Clark M, eds. Clinical medicine,6thedition. Philadelphia: SAUNDERS 2005:1163-73.

3. Weir CJ, Muir K, Grosset DG et al. Poor accuracy of stroke scoring systems for differential clinical diagnosis of intracranial haemorrhage and infarction. The Lancet 1994;344:999-1002.

4. Gomes MDM, Costa MF, André C et al. Emergency physician's diagnosis of stroke subtype; an accuracy study. Arq Neuropsiquiatr 1998;56(3-B):523-27.

5. Sotaniemi K.A, Pyhtinen J, Myllyla VV et al. Correlation of Clinical and Computed Tomographic Findings in Stroke Patients. Stroke 1990;21:1562-1566.

6. Mullins ME, Lev MH, Schellingerhout $D$ ey al. Influence of availability of clinical history on detection of early stroke using unenhanced ct and diffusion-weighted MR imaging. AJR 2002;179:223-28.

7. Mohr JP, Foulkes MA, Polis AT et al. Infarct topography and hemiparesis profiles with cerebral convexity infarction: the Stroke Data Bank. Journal of Neurology, Neurosurgery, and Psychiatry 1993;56:344-51. 
8. Whiteley WN, Wardlaw JM, Dennis MS et al. Clinical scores for the identification of stroke and transient ischaemic attack in the emergency department: a cross-sectional study J Neurol Neurosurg Psychiatry. doi:10.1136/jnnp.2010.235010.available at: jnnp.bmj.com access on March 25, 2011

9. Baloch $\mathrm{GH}$, Shaikh S, Jaffery MH et al. Stroke Localization: Clinical Correlation versus Findings of CT Scan Brain in Patients Admitted at Liaquat University Hospital Hyderabad/ Jamshoro JLUMHS 2009; 8: 3-7.

10. Pratheepan GJ, de Silva DBYN, Weerarathna TP et al. Value of CT scanning in the diagnosis and management of patients admitted with stroke to a tertiary care center in Sri Lanka. Galle Medical Journal 2008; 13:1-6.

11. Khan J and Rehman A. Comparison of clinical diagnosis with computed tomography in ascertaining type of stroke. J Ayub Medical Abbottabad 2005;17(3): 65-7.

12. Siddique MAN, Nur Z, Mahbub MS et al. Clinical presentation and epidemiology of stroke -a study of 100 cases. J Medicine 2009; 10: 86-9.

13. Hill MD. Specific clinical findings, including coma, neck stiffness and seizures, increase the likelihood of haemorrhagic stroke, but no combination of features is definitively
diagnostic.Evidence-Based Medicine December 2010;15:183-84.

14. Wardlaw JM. Is routine computed tomography in strokes unnecessary? BMJ 1994;309: 1498-99.

15. Naik M, Rauniyar RK, Sharma UK et al. Clinico-radiological profile of stroke in eastern Nepal: A computed tomographic study Kathmandu University Medical Journal 2006; 4: 161-66.

16. Poungvarin N, Viriyavejakul A, Komontri C. Siriraj Stroke Score and validation study to distinguish supratentorial intracerebral hemorrhage from infarction. BMJ 1991; 302:1565-67.

17. Kochar DK, Joshi A, Agarwal N, Aseri S, Sharma BV, Agarwal TD. Poor diagnostic accuracy and applicability of Siriraj Stroke Score, Allen score and their combination in differentiating acute haemorrhagic and thrombotic stroke. J Assoc Physicians India 2000;48:584-88.

18. Shah FU, Salih M, Saeed MA, Tariq M. Validity of Siriraj Stroke Scoring. J Coll Physicians Surg Pak 2003;13:391-93.

19. Nor AM, Davis J, Sen B, et al. The Recognition of Stroke in the Emergency Room (ROSIER) scale: development and validation of a stroke recognition instrument. Lancet Neurol 2005;4:727-34. 